\title{
Effects of Intravitreal Injection on Ocular Surface and Anterior Segment Parameters
}

\author{
(D) Hafize Gokben Ulutas, (D) Neslihan Parmak Yener \\ Department of Opthalmology, University of Health Sciences Bursa Yuksek Ihtisas Training and Research Hospital, Bursa, Turkey
}

\begin{abstract}
Objectives: This study was designed to investigate the long-term effects of intravitreal anti-vascular endothelial growth factor (VEGF) injections on ocular surface and anterior segment parameters. A comparison was performed of the treated eye and the fellow healthy eye.

Methods: The study group included patients who had received at least 3 intravitreal injections of anti-VEGF agents (bevacizumab, ranibizumab, or aflibercept) for retinal vascular disorders. All of the subjects were treated in only I eye. A complete ophthalmological examination, including evaluation of tear break-up time (TBUT), the Schirmer I test, the Oxford Grading Scale, and the Ocular Surface Disease Index (OSDI) questionnaire, corneal topography, and specular microscopy were performed in both eyes I month after the last injection.

Results: In this study, 49 eyes of 49 patients who underwent regular intravitreal injection in I eye were enrolled. The mean age was $63.85 \pm 9.8$ years (range: $29-86$ years). A mean of $4.06 \pm 1.7$ (range: $3-\mathrm{I}$ I) injections were administered. There was no significant difference in the intraocular pressure, TBUT, Schirmer I, fluorescein staining, or specular microscopy parameters ( $p>0.05)$. The mean non-invasive TBUT first and average values; the central, thinnest, and apex corneal thickness measurements; the anterior chamber depth, irido-corneal angle, corneal volume, and keratometry values were similar in each individual ( $p>0.05$ for all parameters). The mean OSDI score was $27.5 \pm 17.6$ for the injected eyes and I5.9 \pm 12.9 for the non-injected eyes $(p<0.000 \mathrm{l})$.

Conclusion: Intravitreal anti-VEGF injections had no effect on ocular surface, corneal endothe-lium, and anterior segment parameters.
\end{abstract}

Keywords: Anterior segment, cornea, corneal topography, intravitreal injection, ocular surface

\section{Introduction}

The use of intravitreal anti-vascular endothelial growth factor (VEGF) agents to treat retinal vascular pathologies and exudative senile macular degeneration has continued to grow. Intravitreal injections are now the most common ophthalmological treatment (I). Millions of intravitreal injections are performed annually to address posterior segment pathologies of the eye. Many studies have demonstrated that intravitreal administration of anti-VEGF agents not only decreased vision loss but even increased vision $(2,3)$. A large number of patients have received monthly injections for many years now.

Intravitreal injections also have several potential adverse effects (subconjunctival hemorrhage, elevation of intraocular

How to cite this article: Gokben Ulutas H, Parmak Yener N. Effects of Intravitreal Injection on Ocular Surface and Anterior Segment Parameters. Beyoglu Eye J 2021; 6(2): 84-89.

Address for correspondence: Hafize Gokben Ulutas, MD. Saglik Bilimleri Universitesi Bursa Yuksek Ihtisas Egitim ve Arastirma Hastanesi, Goz Hastaliklari Anabilim Dali, Bursa, Turkey

Phone: +90 5056058585 E-mail: gokbenbilekulutas@gmail.com

Submitted Date: December 28, 2020 Accepted Date: April 25 2021, 2020 Available Online Date: June 08,2021

${ }^{\circ}$ Copyright 2021 by Beyoglu Eye Training and Research Hospital - Available online at www.beyoglueye.com OPEN ACCESS This work is licensed under a Creative Commons Attribution-NonCommercial 4.0 International License. 
pressure [IOP], etc.). The most destructive and feared side effect is endophthalmitis (4). Numerous measures are taken to reduce the risk of endophthalmitis, such as using povidone iodine, which provides highly effective periocular and ocular surface sterilization, and applying antibiotic prophylaxis post injection. However, it has been reported in the literature that the application of $5 \%$ povidone iodine to the ocular surface can disrupt the epithelial integrity of the cornea and conjunctiva and cause complaints of visual impairment, burning, or stinging (5). The use of prophylactic antibiotics containing preservatives after intravitreal injection can also lead to ocular surface irritation and corneal and conjunctival tissue damage (6). Eye drops with preservatives applied once a day for 7 days alter the ocular surface and may cause dry eye symptoms (7).

Dry eye is a multifactorial disease of the tear film and the ocular surface, which causes visual impairment and eye discomfort. Tear instability increases the risk factors associated with ocular surface disease. Dry eye is accompanied by increased osmolarity of the tear film and inflammation of the ocular surface (8). These symptoms are often exacerbated by activities such as reading, digital screen exposure, and driving, particularly at night. Most of the symptoms gets worse with age (9).

Elevated inflammatory markers, like interleukin la, tumor necrosis factor alpha, and VEGF, have been associated with dry eye syndrome. Epithelial cell damage and apoptosis of goblet cells occur as a result of inflammation. Mucus secretion decreases, and the ocular surface becomes vulnerable to chemical and physical damage $(10, \mathrm{I})$. Dry eye disease can also be exacerbated by intravitreal injections through the same paths. It is one of the most common ocular side effects of intravitreal injections and yet is often overlooked. Many patients undergoing intravitreal treatment are already predisposed to dry eye disease due to their age or systemic disorders.

Several studies have investigated the effect of intravitreal injections on the ocular surface in the early period (I2). This study was designed to examine the long-term effects of intravitreal anti-VEGF injections on ocular surface and anterior segment parameters by comparing a treated eye and a healthy eye in the same patient.

\section{Methods}

This study was approved by the clinical research ethics committee of the hospital on March 27, 2019 (no: 20I I-KAEK25 2019/03-24). The research was conducted in the Department of Ophthalmology between April 2019 and January 2020 and the study protocols adhered to the tenets of the Declaration of Helsinki. Written, informed consent was provided by all of the participants.
Patients who had received at least 3 intravitreal injections of anti-VEGF agents (bevacizumab, ranibizumab, or aflibercept) in just I eye for diabetic macular edema (DME), choroidal neovascularization (CNV), or retinal venous occlusion (RVO) were included in the study. Exclusion criteria were prior ocular surface surgery (pterygium, refractive surgery other than cataract), use of contact lenses, or a history of systemic or dermatological diseases that might affect the ocular surface, ocular surface burn, or regular treatment for glaucoma.

\section{Injection Technique}

All of the injections were administered after topical anesthesia with proparacaine (Alcaine 0.5\%; Alcon AG, Geneva, Switzerland). The eyelid and periocular surface was disinfected with $10 \%$ povidone iodine and covered with an ophthalmic surgical drape. Next, $5 \%$ povidone iodine was instilled in the cornea, conjunctiva, and palpebral fornixes, and left in place for 2 minutes before washing it out with a balanced sterile saline solution. The anti-VEGF was inject-ed intravitreally $3.5 \mathrm{~mm}$ from the limbus into the superotemporal quadrant using a $30-\mathrm{G}$ needle. Post-injection light perception was assessed and the eye was then maintained in a closed position for 4 hours. Finally, topical moxifloxacin hydrochloride (Vigamox; Novartis International AG, Basel, Switzerland) was administered 5 times a day for 5 days.

All of the patients underwent a complete ophthalmological examination after at least 3 intravitreal anti-VEGF injections. The exam included measurement of refraction (RK-F2 Full Au-to Ref-Keratometer; Canon Medical Systems Corp., Otawara, Tochigi, Japan), best corrected visual acuity, biomicroscopy, non-contact tonometry, and corneal pachymetry (CT-IP; Topcon Positioning Systems Inc., Tokyo, Japan), optical coherence tomography (iVue; Optovue Inc., Fremont, CA, USA), corneal topography (Sirius; Costruzioni Strumenti Oftalmici SRL, Florence, Italy), specular microscopy (NonconRobo NSP-9900; Konan Medical Inc., Nishinomiya, Hyogo, Japan) and a dilated fundus examination I month after the last injection. Fundus fluorescein angiography was performed at the initial postprocedural examination for all of the patients.

The severity of the dry eye symptoms was measured using the Ocular Surface Disease Index (OSDI) questionnaire. The patients were asked to score both the injected and the fellow eye. The OSDI is evaluated on a scale from 0 to 100; a higher score indicates more severe disease. The Schirmer I test, TBUT assessment, and fluorescein staining were performed on both eyes simultaneously. Standard Schirmer strips were left in place for 5 minutes or until they were completely saturated with tears. The wetness of the strips was measured according to the millimeter markings provided on the strip. The TBUT was determined using the time until the first dry spots appeared on the cornea after the instillation of $0.5 \%$ topical fluorescein. The fluorescein 
staining method used was identical to that used in the TBUT test. The corneal punctate staining was graded on the Oxford Grading Scale (range: 0-5) (13).

\section{Corneal Topography}

The parameters of the anterior segment and non-invasive tear film break time (NITFBUT) were evaluated using the Phoenix tear film imaging module and the Sirius corneal topography device, which has a Scheimpflug camera system. The best alignment and fixation data were used for further analysis. The corneal thickness and refractive map indices were evaluated using the most appropriate sphere for each patient. The mean keratometry front $(\mathrm{Km})$ and back surface of the cornea, apical keratometry (Kmax), corneal volume (CV), horizontal visible iris diameter (HVID), anterior chamber volume $(A C V)$, anterior chamber depth $(A C D)$, irido-corneal angle (ICA), central corneal thickness (CCT), thinnest corneal thickness (TCT), and apical corneal thickness (ACT) values were recorded. The patients were asked to blink twice and maintain the eye open during imaging. The maximum duration of imaging was 17 seconds, either due to blinking or disruption of the corneal surface. The first and average NITFBUT time per image were noted.

\section{Specular Microscopy}

Non-contact specular microscopy was used to obtain 3 measurements from the center of the cornea of each patient. At least 100 adjacent cells were analyzed and the endothelial cell density (CD), coefficient of variation (CoV) and cell hexagonal percentage (Hex) were calculated automatically.

\section{Statistical Analysis}

The Shapiro-Wilk test was used to assess the normality of the data distribution. Continuous and categorical variables were presented as mean $\pm S D$ and frequency, and number and percentage, respectively. A paired samples t-test and the Wilcoxon signed rank test were used for comparison of the intravitreal injection eyes, and the fellow eyes constituted a control. Pearson and Spearman correlation coefficients were used to determine the relationship between variables. The accepted level of statistical significance was $\alpha=0.05$. All of the statistical analyses were performed with IBM SPSS Statistics for Windows, Version 23.0 (IBM Corp., Armonk, NY, USA).

\section{Results}

In all, 49 eyes of 49 patients were enrolled in this study; regular intravitreal injection was performed in I eye and the untreated fellow eye was used as a control. The mean age of the participants was 63.85 \pm 9.8 years (range: 29-86 years). Twenty-five patients (5I.0\%) had CNV, I5 (30.6\%) had RVO, and 9 (18.3\%) were diagnosed with DME. The group comprised 25 male subjects (5I\%) and 24 female subjects (49\%). A mean of $4.06 \pm 1.7$ injections (range: $3-11$ injections) were administered. Demographic features and details of the diagnosis, treatment, and injections are summarized in Table I.

The distribution of the data before and after injections was homogeneous. There was no statistically significant difference between the 2 eyes in the IOP, TBUT, Schirmer I, fluorescein staining, or specular microscopy (CD, CoV, Hex, and pachymeter) parameters ( $p>0.05)$. The mean OSDI score was $27.5 \pm 17.6$ for the injected eyes and $15.9 \pm 12.9$ for the untreated eyes. The OSDI score was significantly higher in the eyes treated with anti-VEGF injections $(p<0.000 \mathrm{I})$. The results of the examinations are presented in Table 2 .

Table I. Demographic features, diagnosis, and treatment

Age (years, range)

$63.85 \pm 9.8(29-86)$

Gender, (n, \%)

Female

Male

Diagnosis, (n, \%)

SMD

DME

RVO

Treatment, (n, \%)

Aflibercept

Ranibuzumab

Bevacizumab

Injections (n)

$4.06 \pm 1.7(3-11)$

DME: Diabetic macular edema; RVO: Retinal vascular occlusion; SMD: Senile macular degeneration.

Table 2. Ocular examination of the injected eye and the fellow eye

\begin{tabular}{lccc} 
& Injected eye & Fellow eye & p \\
\hline IOP $(\mathrm{mmHg})$ & $17.9 \pm 3.5$ & $17.7 \pm 2.8$ & 0.816 \\
Pachymeter $(\mu \mathrm{m})$ & $546 \pm 32.7$ & $547 \pm 31.3$ & 0.889 \\
OSDI score & $27.5 \pm 17.6$ & $15.9 \pm 12.9$ & $<0.001$ \\
TBUT $(\mathrm{s})$ & $10.8 \pm 4.2$ & $11.4 \pm 3.9$ & 0.102 \\
Schirmer I $(\mathrm{mm})$ & $13.2 \pm 7$ & $14 \pm 8.2$ & 0.406 \\
Oxford Grading Scale & $0.45 \pm 0.7$ & $0.64 \pm 0.7$ & 0.097 \\
Specular Microscopy & & & \\
$\quad$ CD (cells/mm $)$ & $2641.3 \pm 296$ & $2636.68 \pm 293$ & 0.908 \\
CoV $(\%)$ & $46 \pm 6$ & $46.3 \pm 7$ & 0.796 \\
Hex $(\%)$ & $42.8 \pm 6$ & $43.1 \pm 8$ & 0.861 \\
Pachymeter $(\mu \mathrm{m})$ & $503.2 \pm 43.9$ & $506.3 \pm 37.5$ & 0.781 \\
\hline
\end{tabular}

The parameters above are presented as mean \pm SD; CD: Cell density; CoV: Coefficient of variation; Hex: Hexagonality; IOP: intraocular pressure; OSDI: Ocular Surface Disease Index;TBUT:Tear break-up time. 
Table 3 illustrates the corneal topographic analysis and anterior chamber parameters of the injected and fellow eyes. The mean first NITFBUT, average NITFBUT, CCT, TCT, ACT, HVID, ACD, ACV, ICA, CV, front $\mathrm{Km}$, back $\mathrm{Km}$, and apical $\mathrm{K}$ values were similar in both eyes ( $p>0.05$ for all parameters).

Parameters were seen to correlate with patient age and gender. The apical $\mathrm{K}$ difference between a healthy and a treated eye increased significantly with age $(r=0.330$; $p=0.038)$. Similarly, the cell density $(C D)$ between healthy and treated eyes also increased with age $(r=0.523 ; p=0.00 \mathrm{I})$. No significant difference was observed between genders in any of the parameters.

The patients were also grouped and assessed based on the diagnosis. In the AMD group, the Schirmer I difference between the healthy and the injected eye decreased with age $(r=-0.427 ; p=0.033)$ and the $C D$ difference increased $(r=0.652 ; p=0.005)$. With additional injections, the ACT difference between eyes decreased $(r=-0.492 ; p=0.028)$ and the CV increased $(r=0.532 ; p=0.016)$.

\section{Discussion}

The results of this analysis of the changes in the ocular surface and anterior segment parameters of patients who had received at least 3 monthly intravitreal injections revealed no statistically significant difference between the healthy and

Table 3. Topographic examination of the injected eye and the fellow eye

\begin{tabular}{lccc} 
& Injected eye & Fellow eye & p \\
\hline NITFBUT first $(s)$ & $9.8 \pm 5.2$ & $10.2 \pm 5.3$ & 0.630 \\
NITFBUT avg $(s)$ & $11.8 \pm 4.3$ & $11.8 \pm 4.5$ & 0.924 \\
CCT $(\mu \mathrm{m})$ & $533.8 \pm 33.1$ & $537.5 \pm 34.5$ & 0.135 \\
TCT $(\mu \mathrm{m})$ & $529.2 \pm 32.6$ & $532.7 \pm 35.6$ & 0.169 \\
ACT $(\mu \mathrm{m})$ & $581.9 \pm 49.9$ & $593.2 \pm 58.1$ & 0.184 \\
HVID $(\mathrm{mm})$ & $11.4 \pm 0.5$ & $11.7 \pm 0.5$ & 0.299 \\
ACD $(\mathrm{mm})$ & $3.1 \pm 0.9$ & $2.9 \pm 0.7$ & 0.715 \\
ACV $\left.(\mathrm{mm})^{3}\right)$ & $125.16 \pm 41.9$ & $121.8 \pm 34.0$ & 0.742 \\
ICA & $45.5 \pm 9.5$ & $44.4 \pm 9.8$ & 0.323 \\
CV & $57.3 \pm 3.5$ & $57.7 \pm 3.6$ & 0.191 \\
Front Km & $43.8 \pm 1.6$ & $43.9 \pm 1.5$ & 0.905 \\
Back Km & $6.3 \pm 0.2$ & $6.3 \pm 0.2$ & 0.323 \\
Apical K & $47.3 \pm 4.4$ & $47.6 \pm 5$ & 0.635 \\
\hline
\end{tabular}

The parameters above are presented as mean \pm SD;Avg:Average; ACD: ACD:Anterior chamber depth;ACT:Apex corneal thickness;ACV:Anterior chamber volume; CCT: Central corneal thickness; $\mathrm{CV}$ : Corneal volume; HVID: Horizontal visible iris diameter; ICA: Irido-corneal angle; K: Corneal curvature; Km: Mean keratometry; NITFBUT: Non-invasive tear film breakup time;TCT:Thinnest corneal thickness. the treated eyes, with the exception of the OSDI score. Responses to OSDI questions about vision reduction and vision blur likely reflected reduced visual acuity in the treated eye. In addition, due to the subjective nature of the OSDI score, participant bias might have been a factor. The lack of change in the Schirmer I test, TBUT, NITFBUT, and Oxford Grading Scale indicated that intravitreal injection did not appear to have an impact on the development of dry eye.

Our review of the literature yielded 3 studies of the ocular surface after intravitreal injection. Saedon et al. (14) found no statistically significant difference in the tear film osmolarity between an injected and a healthy eye; however, the Schien Dry Eye Questionnaire and Oxford Grading Scale results were statistically significantly different. Dry eye was attributed to repeated intravitreal injections. The mean patient age (79 years) and number of injections number (10.4) in that study were higher than those of our study. We observed that the Schirmer I test difference between healthy and injected eyes decreased with age in the AMD group $(r=-0.427$; $p=0.033)$. Srinagesh et al. (I5) noted in a review performed 2 to 4 weeks after intravitreal injection that the injected eye had a significantly higher OSDI score and greater fluorescein staining. They did not detect any difference in the Schirmer I test or TBUT values, similar to our findings; however, the small number of patients and the initiation of examinations at the second week may be noteworthy. Dohlman et al. (I2) found that corneal epitheliopathy developed after intravitreal injection (fluorescein staining score), and that ocular surface disorder symptoms occurred (Symptom Assessment Questionnaire in Dry Eye), due to the povidone iodine used at the time of the injections, but did not persist long term.

After ocular surgical treatment, a number of patients experience postoperative discomfort and grittiness of the eye. These symptoms may develop due to epithelial damage due to eye irrigation during surgery, increased secretion of inflammatory cytokines in tears, decreased corneal sensitivity, the use of topical anesthetic drops and topical eye drops containing preservatives after surgery $(16,17)$. In our clinical experience, patients have developed burning, stinging, and foreign body sensations in the first days after an intravitreal injection. However, in this study, we found that intravitreal injection did not cause clinical discomfort of the ocular surface during long-term follow-up. This was an expected result, since adjuvant treatment with povidone iodine and topical antibiotic eye drops in the early period has been acknowledged in the literature to be the primary source of ocular surface discomfort $(5,16,17)$.

It would appear from our results that the OSDI score, the only parameter that demonstrated a significant difference, should not be considered in isolation while evaluating these patients. Although the OSDI score is an established 
and reliable measure, additional inconsistent and subjective measurements resulting from variations regarding ocular surface symptoms, visual status, and environmental factors in this questionnaire should also be taken into account.

The potential long-term impact of anti-VEGF agents not only on the ocular surface, but also on the whole-body system is an exciting area of investigation. Researchers continue to examine the transition pathways of these agents through the systemic circulation and anterior segment structures to further understand the possible side effects of these agents. We analyzed potential effects on the ocular surface and anterior segment measurements using several imaging techniques and observed no specific side-effects caused by these agents.

The underlying conditions of older patients, such as diabetes, hypertension, and hormonal changes (menopause), can further contribute to an increased risk of dry eye and ocular surface disease (18). This makes it difficult to distinguish the role of intravitreal injections from the effect of systemic diseases. In our study, the apical $K$ and $C D$ difference between healthy and treated eyes was significantly higher as the patient age increased $(r=0.330 ; p=0.038$ and $r=0.523 ; p=0.001$, respectively). Studies that have examined endothelial changes before and after intravitreal injection have found that anti-VEGF agents did not cause alteration of the corneal endothelium (19-2I). We also observed no endothelial changes between the healthy eye and the treated eye. However, with aging, the difference in CD between the eyes increases. It has been hypothesized that a decrease in endothelial cells with aging and subsequent reduced ability to repair itself creates more sensitivity to eye interventions (22). In this study, no significant difference was seen in any of the anterior chamber parameters (CCT, TCT, ACT, HVID, $\mathrm{ACD}, \mathrm{ACV}, \mathrm{ICA}, \mathrm{CV}$, front $\mathrm{Km}$, back $\mathrm{Km}$ and apical $\mathrm{K}$ ). As a result, we concluded that intravitreal injections did not have a long-term effect on the anterior chamber parameters and the cornea. Kerimoğlu et al. (23) reported a brief statistically significant narrowing in anterior chamber depth and volume after intravitreal injection and a significant increase in IOP. Omay et al. (24) investigated the effects of intravitreal bevacizumab and ranibizumab injections on CCT and IOP at the Ist, 7th, and 30th day post intravitreal injection and observed a hyper-acute increase in IOP, but noted that the effect was generally reversible. To the best of our knowledge, our study is the first to look at long-term effects of intravitreal injections on ACD and ICA. It has been shown that an increase in IOP and alterations in aqueous pressure gradients between the anterior and posterior chambers caused by the amount of treatment agent brought into the eye globe can cause immediate forward displacement of the iris root, lens, and zonular fibers, and consequent narrowing of the ACD and ICA. However, these alterations are transient; during the elimination of the anti-VEGF agent, the structures typically return to their original positions and the anterior segment dynamics normalize over time (23-25).

There are several limitations to this study. First, the small sample size restricts generalization to a larger population. Second, the OSDI score is a subjective measure of the ocular surface. We elected to combine this questionnaire with other ocular surface measurements in an effort to address this weakness. Finally, to better understand the main effects of anti-VEGF agents on the cornea and the anterior segment, in vivo examination of the aqueous samples in the chronic period would have been optimal, however, this was not ethically possible.

In conclusion, our findings indicated that intravitreal antiVEGF injections had no effect on ocular surface and anterior segment parameters in long-term follow-up. A study with a larger patient group and a longer period of follow-up would be useful to further evaluate these results.

\section{Disclosures}

Ethics Committee Approval: This study was approved by the clinical research ethics committee of the hospital on March 27, 2019 (no: 20II-KAEK-25 2019/03-24).

Peer-review: Externally peer-reviewed.

Conflict of Interest: None declared.

Authorship Contributions: Involved in design and conduct of the study (HGU, NPY); preparation and review of the study (HGU); data collection (HGU, NPY); and statistical analysis (HGU).

\section{References}

I. Campell RJ, Bronskill SE, Bell CM, Paterson MJ, Whitehead M, Gill SS. Rapid expansion of intravitreal drug injection procedures, 2000 to 2008: a population-based analysis. Arch Ophthalmol 2010;128:359-62. [CrossRef]

2. Rosenfeld PJ, Brown DM, Heier JS, Boyer DS, Kaiser PK, Chung CY, et al; MARINA Study Group. Ranibizumab for neovascular age related macular degeneration. N Engl J Med 2006;355: 14 I 9 3I. [CrossRef]

3. Brown DM, Kaiser PK, Michels M, Soubrane G, Heier JS, Kim RY, et al. ANCHOR Study Group. Ranibizumab versus verteporfirin for neovascular age-related macular degeneration. N Engl J Med 2006;355: 1432-44. [CrossRef]

4. Falavarjani KG, QD Nguyen. Adverse events and complications associated with in-travitreal injection of anti-VEGF agents: a review of literature. Eye 2013;27:787-94. [CrossRef]

5. Ridder WH, Oquindo C, Dhamdhere K, Burke J. Effect of povidone iodine $5 \%$ on the cornea, vision, and subjective comfort. Optom Vis Sci 2017;94:732-4I. [CrossRef]

6. Baudouin C, Labbe A, Liang H, Pauly A, Brignole-Baudouin F. Preservatives in eyedrops: 400 The good, the bad and the ugly. Prog Retin Eye Res 2010;29:3 12-34. [CrossRef]

7. Zhang R, Park M, Richardson A, Tedla N, Pandzic E, Paiva CS, et 
al. Dose-dependent benzalkonium chloride toxicity imparts ocular surface epithelial changes with features of dry eye disease. Ocul Surf 2020; 18:158-69. [CrossRef]

8. The definition and classification of dry eye disease: report of the Definition and Classification Subcommittee of the International Dry Eye Workshop (2007). Ocul Surf 2007;5:75-92. [CrossRef]

9. Miljanovic B, Dana R. Impact of dry eye syndrome on vision-related quality of life. Am J Ophthalmol 2007; I43:409-15. [CrossRef]

10. Yeh S, Song XJ, Farley W, Li DQ, Stern ME, Pflugfelder SC. Apoptosis of ocular surface cells in experimentally induced dry eye. Invest Ophthalmol Vis Sci 2003;44: I24-9. [CrossRef]

II. Calonge M, Salamanca EA, Diebold Y, Garcia MJG, Reinoso R, Herreras JM, et al. Dry eye disease as an inflammatory disorder. Ocul Immunol Inflamm 2010; 18:244-53. [CrossRef]

12. Dohlman TH, Lertsuwanroj B, D'Amico DJ, Ciralsky JB, Kiss S. Evaluation of signs and symptoms of ocular surface disease after intravitreal injection. Acta Ophthalmol 2019;97:I I54-6. [CrossRef]

13. Bron AJ, Evans VE, Smith JA. Grading of corneal and conjunctival staining in the context of other dry eye tests. Cornea 2003;22:640-50. [CrossRef]

14. Seadon H, Nosek J, Phillips J, Narendran N, Yang YC. Ocular surface effects of repeated application of povidone iodine in patients receiving frequent intravitreal injections. Cutan Ocul Toxicol 2017;36:343-6. [CrossRef]

15. Srinagesh V, Ellenberg D, Scharper PH, Etter J. Intravitreal dry eye study. Invest Ophthalmol Vis Sci 20I4;55:3696-6.

16. Li XM, Hu L, Hu J, Wang W. Investigation of dry eye disease and analysis of the pathogenic factors in patients after cataract surgery. Cornea 2007;26:16-20. [CrossRef]

17. Simone JN, Whitacre MM. Effects of anti-inflammatory drugs following cataract extraction. Curr Opin Ophthalmol
200I;12:63-7. [CrossRef]

18. Özcura F, Aydin S, Helvaci MR. Ocular Surface Disease Index for the Diagnosis of Dry Eye Syndrome. Ocular Immunology and Inflammation 2007; 15:389-93. [CrossRef]

19. Guzel H, Bakbak B, Koylu MT, Gonul S, Ozturk B, Gedik S. The effect and safety of intravitreal injection of ranibizumab and bevacizumab on the cornea endothelium in the treatment of diabetic macular edema. Cutan Ocu Toxicol 2017;36:5-8. [CrossRef]

20. Arslan GD, Guven D, Alkan AA, Kacar H, Demir M. Short-term effects of intravitreal anti-vascular endothelial growth factor agents on cornea, anterior chamber, and intraocular pressure. Cutan Ocul Toxicol 2019;38:344-8. [CrossRef]

21. Lass JH, Benetz BA, Menegay HJ, Tisipis CP, Cook JC, Boyer DS, et al. Effects of repeated intravitreal aflibercept injection on the corneal endothelium in patients with age-related macular degeneration: Outcomes from the RE-VIEW Study. Cornea 2018;37:596-60I. [CrossRef]

22. Faragher RGA, Mulholland B, Sandeman S, Khaw P. Aging and the cornea. Br J Ophthalmol 1997;81:814-7. [CrossRef]

23. Kerimoglu H, Ozturk BT, Bozkurt B, Okka M, OKudan S. Does lens status affect the course of early intraocular pressure and anterior chamber changes after intravitreal injection? Acta Ophthalmol 201 1;89:138-42. [CrossRef]

24. Omay E, Elgin U, Sen E, Yılmazbaş P. The early effects of intravitreal anti vascular endothelial growth factor agents on intraocular pressure and central corneal thickness. International Ophthalmology 2016;36:665-70. [CrossRef]

25. Alkin Z, Perente I, Altan C, Konstantinidis A, Ozkaya A, Yuksel $K$, et al. Changes in anterior segment morphology after intravitreal injection of bevacizumab, and bevacizumab-triamcinolone acetate combination. Eur J Ophthalmol 2013;23:504-9. [CrossRef] 